\title{
Necrotizing Keratitis after Laser Refractive Surgery in Patients with Inactive Inflammatory Bowel Disease
}

\author{
Muhammad Aman-Ullah ${ }^{a}$ Howard V. Gimbel ${ }^{a, b}$ \\ Mona K. Purba ${ }^{a}$ John A. van Westenbrugge ${ }^{a}$ \\ ${ }^{a}$ Gimbel Eye Centre, Calgary, Alta., Canada; ${ }^{b}$ Loma Linda University, \\ Loma Linda, Calif., USA
}

\section{Key Words}

Keratitis - Inflammatory bowel disease - Photorefractive keratectomy · Laser-assisted in situ keratomileusis

\begin{abstract}
Two cases of necrotizing keratitis following laser refractive corneal surgery, with stable and controlled Crohn's disease are described. A 40-year-old woman developed bilateral stromal inflammation and inferior thinning in the right eye along the flap edge within 1 day of uneventful bilateral IntraLase laser-assisted in situ keratomileusis. The other case is a 30year-old man who also developed bilateral inferior stromal infiltrates 2 days following photorefractive keratectomy. Both cases were aggressively treated with systemic and topical corticosteroids. The infiltrates in both patients gradually resolved, with one relapse during the 7 months period of follow-up in the first case. These cases highlight the importance of taking precautions considering this and similar autoimmune conditions as a relative contraindication to refractive surgery.
\end{abstract}

\section{Introduction}

Inflammatory bowel diseases (IBD) include ulcerative colitis (UC) and Crohn's disease (CD). These are chronic inflammatory conditions affecting the gastrointestinal tract characterized by the presence of extensive ulcerations in the intestines. The pathogenesis of IBD is hypothesized to involve an abnormal immune response to environmental antigens in a person who is already genetically predisposed. A vast majority of these patients develop intestinal complications, with both high incidences of endoscopic recurrence and symptomatic recurrence. It is reported that ocular 
surgery in IBD and other autoimmune disorders can trigger ocular complications, such as corneal infiltrates, ulcerations and necrotizing inflammations [1,2]. Other ocular complications include scleritis, episcleritis, ulcerative keratitis, corneal infiltrates, uveitis, vasculitis, and optic neuritis. A few studies have demonstrated that laserassisted in situ keratomileusis (LASIK) can be performed effectively and safely in selected patients with stable and controlled IBD, including CD [3, 4]. Successful treatments of refractive conditions for patients with IBD and other autoimmune conditions using photorefractive keratectomy (PRK) and IntraLase LASIK have been reported, with a postoperative corneal complication rate no greater than for patients without these conditions. Due to the potential for ocular complications in some patients, IBD and other autoimmune conditions are considered to be a relative contraindication to laser refractive surgery.

In this paper, we describe two case reports of PRK and IntraLase LASIK procedures in individuals with IBD in long-term remission that developed infiltrates post IntraLase LASIK in the first case and necrotizing keratitis along the flap incision site post PRK in the second case.

\section{Case Report 1}

A 40-year-old woman presented for refractive surgery assessment at the Gimbel Eye Centre, Calgary, Canada, in May 2010. The patient had worn contact lenses for the last 22 years and now was becoming intolerant due to dryness. She had CD, which had been in remission for the last 3 years. She was not on any medications and denied having any ocular pathology in the past. A complete eye examination was performed to screen for any ocular pathology and determine her candidacy for refractive surgery.

The manifest refraction was $-3.75-0.75 \times 121$ in the right eye and $-4.25-0.25 \times 177$ in the left eye, achieving a corrected distance visual acuity of $20 / 20$ in both eyes. Keratometry (diopters) was 44.75 @ 149/45.50@ 59 in the right eye and $45.0 @ 180 / 45.50 @ 90$ in the left eye. The central corneal thickness was $537 \mu \mathrm{m}$ in the right eye and $522 \mu \mathrm{m}$ in the left eye. The patient was considered suitable for IntraLase LASIK, targeting both eyes for distance.

An IntraLase FS laser (Abbot Medical Optics, Abbott Park, Ill., USA) was used to create corneal flaps with a depth of $120 \mu \mathrm{m}$ and diameter of $8.8 \mathrm{~mm}$ with a superior hinge, sequentially in both eyes. The ablation was then performed using a wave-front optimized aspheric transition zone ablation pattern with NIDEK EC 5000 CX3 excimer laser (NIDEK, Gamagori, Japan) with a treatment zone of 5 and $9 \mathrm{~mm}$ transition zone. The target refraction was emmetropia in both eyes. The IntraLase LASIK procedure was uneventful in both eyes.

She presented the next day with symmetrical corneal infiltrates at the flap edge at 10 and 2 o'clock, $1 \mathrm{~mm}$ in diameter, surrounding trace inflammatory cells and no overlying epithelial defects. She was started on prednisolone (Pred Forte) 1.0\% ophthalmic solution every hour and moxifloxacin $\mathrm{HCl}$ (Vigamox) $0.5 \%$ six times per day in both eyes. Over the next few days, she was monitored closely and the corneal lesions appeared to be resolving. Her uncorrected visual acuity (UCVA) fluctuated between 20/15 and 20/25 in both eyes, as expected from early IntraLase LASIK healing. One week postoperatively, she had deterioration in the clinical appearance of her corneas. A slit lamp examination showed new infiltrates at the flap edge inferiorly in the right eye ( $\underline{\text { fig. }} \mathbf{1}$ ). The left eye also showed new infiltrates superiorly. A clear interval was seen between the infiltrates and the limbus, and the center in both eyes. The interface remained clear.

The patient's topical treatment was changed to preservative free prednisolone sodium phosphate $0.5 \%$ drops hourly, and Vigamox $0.5 \%$ was continued at six times per day. In the absence of any clinical signs of infective keratitis, autoimmune-related necrotizing keratitis was diagnosed in both 
eyes. Oral prednisolone $80 \mathrm{mg}$ daily, omiprazole $20 \mathrm{mg}$ daily and doxycycline $100 \mathrm{mg}$ twice a day, were also started.

The patient was monitored on a daily basis. The systemic and topical treatment led to a significant improvement, with gradual resolution of flap infiltrates over the next 4 weeks. There remained a faint haze at the site of the original infiltrates. The topical steroid preparation was tapered over the next 4 weeks, and the oral steroid was tapered by $5 \mathrm{mg}$ every 1 week.

At 2 months follow-up, five new infiltrates, each $1 \mathrm{~mm}$ in diameter were noticed at the right eye flap hinge. No overlying epithelial defect or thinning was seen. Tobramycine $0.3 \% /$ dexamethasone $0.1 \%$ (Tobradex) eye drops were started in the right eye. The patient was advised to continue with oral prednisolone $10 \mathrm{mg}$ daily, omiprazole $20 \mathrm{mg}$ daily and doxycycline $100 \mathrm{mg}$ daily. The recurrent infiltrate in the right eye gradually cleared over the next week. The Tobradex $0.3 \%$ eye drops were tapered off gradually for the next 2 weeks, and oral medications were also gradually tapered and stopped in the next 4 weeks.

Seven months postoperatively, the UCVA was $20 / 15^{-2}$ in both eyes, and the manifest refraction was +0.25 spherical in both eyes. A slit lamp examination showed an area of subtle sub-epithelial haze at the temporal aspect of the flap incision (fig. 2). No residual haze or corneal defects were seen in the left eye.

\section{Case Report 2}

A 30-year-old man referred by his optometrist due to his inability to wear contact lenses following an episode 1 year before of infiltrates in the right cornea and peripheral corneal ulcer in the left eye. The patient was not happy with his glasses wear, as he could not wear contact lenses due to dryness and risk of recurrent corneal infection. He had CD, which was in remission with no systemic complaints and no medications for the past 10 years. An eye examination revealed bilateral faint old scars in the inferior peripheral corneas.

The manifest refraction was $-7.00-0.25 \times 96$ in the right eye and $-7.75-0.25 \times 52$ in the left eye, achieving a corrected distance visual acuity of 20/15 in both eyes. Keratometry (diopters) was 44.25 @ 113/44.50@23 in the right eye and $44.75 @ 70 / 45.00 @ 160$ in the left eye. The central corneal thickness was $571 \mu \mathrm{m}$ in the right eye and $576 \mu \mathrm{m}$ in the left eye. Detailed pre operative discussion about the risk of recurrent corneal inflammation with a corneal laser refractive procedure, i.e. PRK and the I LASIK keratomileusis was undertaken. He was also given the choice of intraocular phakic corrective lenses. However, the patient opted for the PRK in view of his active contact sport activities.

PRK surface ablation was performed sequentially, using optimized aspheric transition zone ablation pattern with NIDEK EC 5000 CX3 excimer laser with a treatment zone of 5 and $8 \mathrm{~mm}$ transition zone. The surface epithelium was removed prior with an Amoils epithelial scrubber (Innovative Excimer Solutions, Toronto, Ont., Canada). The target refraction was emmetropia in both eyes. The PRK procedure was uneventful in both eyes.

He presented the second day with inferior corneal infiltrates, near the limbus at 5:30 and 7 o'clock, in the right and left eye, respectively (fig. 3). He was started on fluorometholone (FML) $0.1 \%$ ophthalmic solution and gatifloxacin (Zymar) $0.3 \%$ every $2 \mathrm{~h}$ in both eyes. His UCVA fluctuated between 20/20 and 20/30 in both eyes, as expected from early PRK healing. One week postoperatively, he had a deterioration of his clinical appearance. A slit lamp examination showed new infiltrates superiorly extending from 10:30 to 12:30 o'clock, $1 \mathrm{~mm}$ from the limbus, and inferior confluent area of epithelial defect from 5 to $8: 30$ o'clock, $1 \mathrm{~mm}$ from the limbus. The left eye also showed new infiltrates at 7 o'clock with overlying epithelial thinning.

The patient's topical treatment was changed to Pred Forte $1.0 \%$ eye drops in the right eye and advised to continue FML $0.1 \%$ four times a day in the left eye. Oral prednisolone $80 \mathrm{mg}$ daily, ranitidine $150 \mathrm{mg}$ twice daily, and doxycycline $100 \mathrm{mg}$ daily were also started. With this treatment regime, the new corneal infiltrates in both eyes gradually resolved in 4 weeks. The oral steroid was gradually tapered off with $5 \mathrm{mg}$ less every fifth day. 
Three months postoperatively, the UCVA was 20/15 in the right eye and 20/25 in the left eye, and the manifest refraction was $+0.25-0.50 \times 87$ and $+2.00-1.00 \times 47$, respectively. A slit lamp examination showed areas of subtle sub-epithelial haze superiorly and inferiorly in the right eye and only inferiorly in the left eye. The patient was advised to continue FML $0.1 \%$ four times a day in both eyes and to have the corneal haze monitored regularly by his local optometrist. In the last ocular exam, at 8 months postoperatively by his optometrist, the UCVA was 20/20 in the right eye and $20 / 20^{-2}$ in the left eye, and the manifest refraction was $+0.25+0.25 \times 90$ and $+1.00-0.75 \times 25$, respectively. The subtle sub-epithelial haze had resolved with faint inferior sub-epithelial scars in both eyes, and the patient was taken off the FML $0.1 \%$ by his optometrist.

\section{Discussion}

UC and CD are classified as IBD, which represents a relative contraindication to refractive corneal surgery.

Successful outcomes without ocular complications post refractive corneal surgery have been reported in the literature in patients with IBD [3, 4]. Our center has encountered two cases of corneal necrotizing keratitis following corneal refractive surgery in patients with IBD. A case of surgically induced corneal necrotizing keratitis following LASIK in a patient with CD has been reported in the literature [5]. Our first case is similar to the reported case, having infiltrates that were well defined and straddling the flap edges. There was also no interface haze or granularity, differentiating it from diffuse lamellar keratitis. At a later stage, there was mild inferior flap margin thinning only in the right eye, but no overlying epithelial ulceration. No conjunctival and episcleral inflammation was noticed in either eye. This could be due to aggressive topical and systemic steroid treatment initiated early, in light of the previously reported case.

IBD presents more frequently in the second and third decades of life. Ocular complications in IBD can be primary or secondary to systemic complications [6]. Episcleritis, scleritis, and uveitis are reported to be the frequent primary complications [7]. In comparison to the spondyloarthropathies, IBD patients were female and had uveitis that was bilateral, posterior, insidious in onset, or chronic in duration; episcleritis, scleritis, and glaucoma are also more common [8]. Other reported primary ocular complications are conjunctivitis, proptosis, idiopathic orbital inflammation, extraocular muscle pareses, myositis, orbital cellulitis, peripheral corneal ulcers, macular edema, central serous retinopathy, retrobulbar neuritis, optic neuropathies, bilateral neuroretinitis with secondary vitritis, subretinal fibrosis and panuveitis syndrome, multifocal choroiditis, and pars planitis [9-12]. Secondary ocular complications in IBD are due to malnutrition and ileal resection. These can result in Candida endophthalmitis (parenteral nutrition), decreased tear formation, night blindness (hypovitaminosis A), and cataract (corticosteroid therapy).

Ocular treatment should be started with simultaneous gastroenterologic consultation. Treatment for anterior uveitis and scleritis includes topical steroids and nonsteroidal anti-inflammatory drugs, if approved by the gastroenterologist. Shortterm, systemic corticosteroids are given for severe inflammation not responding to the topical steroids. For posterior uveitis or cystoid macular edema, intravitreal steroids or a fluocinolone acetonide implant can also be used $[13,14]$. In resistant cases with persistent inflammation, cytotoxic immunosuppressive agents (hydroxychloroquine, 
cyclophosphamide, and azathioprine) [15] and cyclosporine can be also considered. In our two cases, we chose to use both topical and systemic corticosteroids with good final visual and structural outcomes.

When making the decision to operate on these patients, one needs to consider the disease process as the two afore-mentioned cases were cases of IBD in remission for several years without any recent flare-ups, yet they both developed features of necrotizing keratitis. For surgeons, who decide to operate on these patients, a careful follow-up is warranted, as these patients may develop sight-threatening complications. In our experience, it was the careful follow-up management, which led to a successful outcome maintaining excellent vision.

In conclusion, both PRK and LASIK can be considered to be reasonable surgical options in selected patients with stable and controlled IBD including CD, resulting in favorable postoperative refractive outcome.

\section{Disclosure Statement}

The authors have no conflicts of interest to declare.
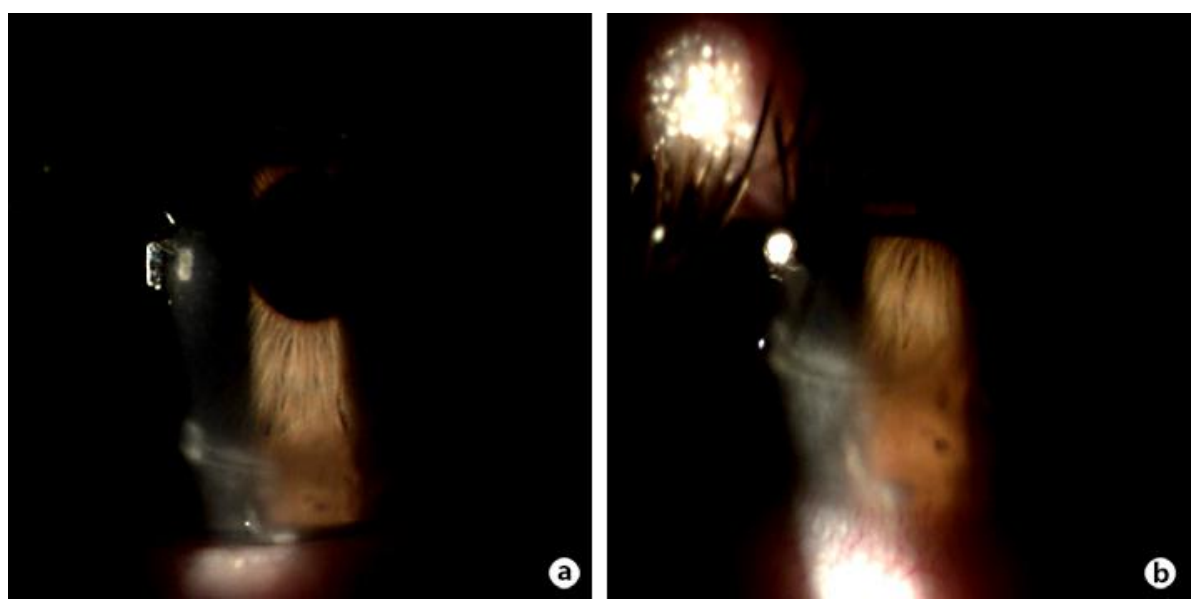

Fig. 1. Slit-lamp photograph of post IntraLase LASIK corneal infiltrates at the flap edge inferiorly of the right eye. a Low magnification. b High magnification. 

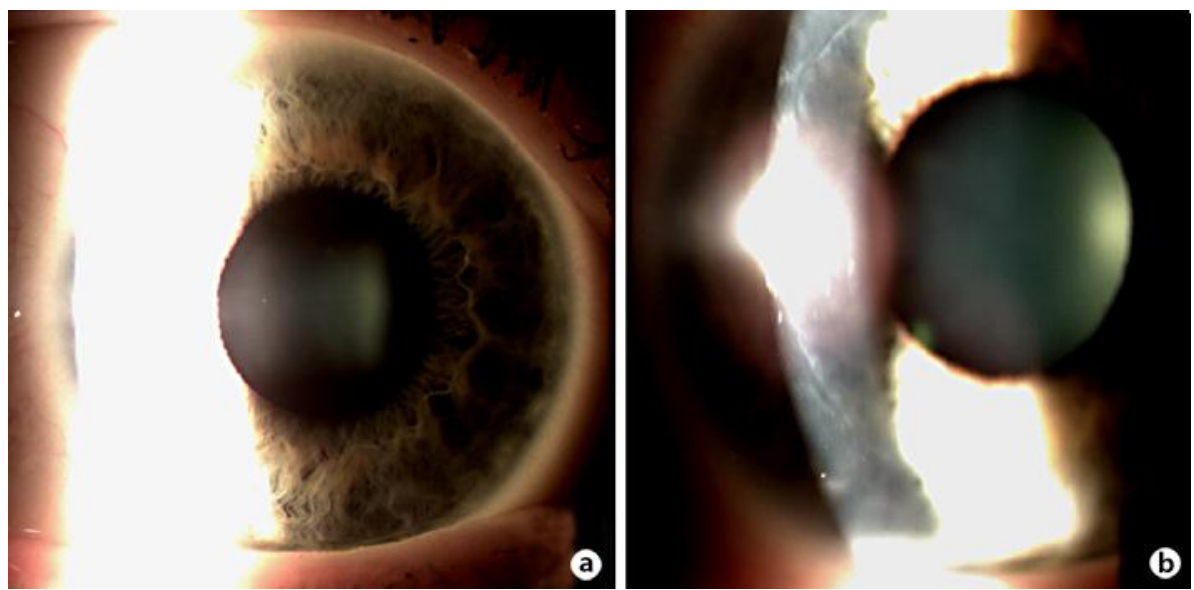

Fig. 2. Slit-lamp photograph of the right eye post treatment at 7 months (a), with subtle sub-epithelial haze at the temporal aspect of flap incision (b).
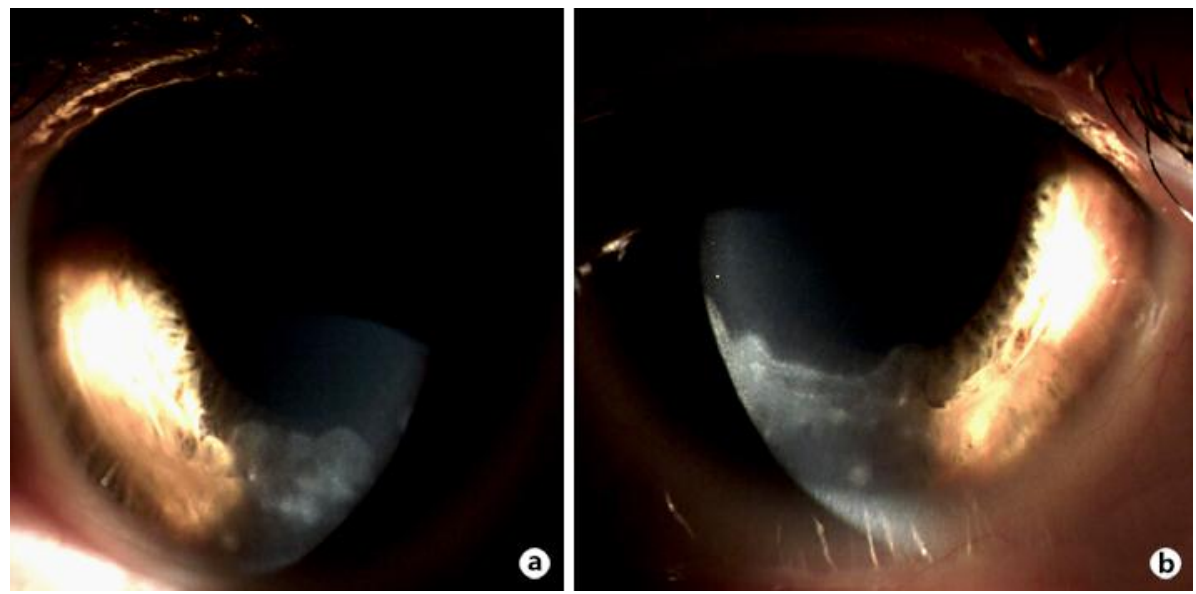

Fig. 3. Slit-lamp photograph of inferior corneal infiltrates post PRK in each eye.

\section{References}

$>1$ Taylor SR, McCluskey P, Lightman S: The ocular manifestations of inflammatory bowel disease. Curr Opin Ophthalmol 2006;17:538-544.

2 Cobo-Soriano R, Beltran J, Baveria J: LASIK outcomes in patients with underlying systemic contraindications; a preliminary study. Ophthalmology 2006;113:1118-1124.

-3 Smith RJ, Maloney RK: Laser in situ keratomileusis in patients with autoimmune diseases. J Cataract Refract Surg 2006;32:1292-1295.

4 Alio JL, Artola A, Belda JI, et al: LASIK in patients with rheumatic diseases; a pilot study. Ophthalmology 2005;112:1948-1954.

5 Carp GI, Verhamme T, Gobbe M, et al: Surgically induced corneal necrotizing keratitis following LASIK in a patient with inflammatory bowel disease. J Cataract Refract Surg 2010;36:1786-1789.

6 Knox DL, Schachat AP, Mustonen E: Primary, secondary, and coincidental ocular complications of Crohn's disease. Ophthalmology 1984;91:163-173. 
7 Ducker JS, Brown GG, Brooks L: Retinal vasculitis in Crohn's disease. Am J Ophthalmol 1987;103:664668.

8 Lyons JL, Rosenbaum JT: Uveitis associated with inflammatory bowel disease compared with uveitis associated with spondyloarthropathy. Arch Ophthalmol 1997;115:61-64.

9 Spraul CW, Buchwald HJ, Lang GK, et al: Recurrent corneal ulcer in a patient with Crohn's disease associated with epidermolysis bullosa acquisita. Klin Monatsbl Augenheilkd 2003;220:423-426.

10 Fuentes-Paez G, Martinez-Osorio H, Herreras JM, et al: Subretinal fibrosis and uveitis syndrome associated with ulcerative colitis. In J Colorectal Dis 2007;22:333-334.

11 Vianna RN, Ozdal PC, Deschenes J: Multifocal choroiditis: an unusual finding in Crohn's disease. Eur J Ophthalmol 2004;14:345-349.

12 Gorrono-Echebarria MB, Albarran F, Marcos A, et al: Inflammatory bowel disease (Crohn's disease) in a Spanish patient with pars plana exudates: report of a new case and review of the literature. Ocul Immunol Inflamm 2002;10:65-68.

13 Jonas JB: Intravitreal triamcinolone acetonide: a change in a paradigm. Ophthalmic Res 2006;38:218245.

14 Jaffe GJ, Martin D, Callanan D, et al: Fluocinolone acetonide implant (Retisert) for noninfectious posterior uveitis: thirty-four-week results of a multicenter randomized clinical study. Ophthalmology 2006;113:1020-1027.

15 Fries W, Giofre MR, Catonoso M, et al: Treatment of acute uveitis with Crohn's disease and sacroileitis with infliximab. Am J Gastroenterol 2002;97:499-500. 